\title{
Segmentation of MRI images to detect multiple sclerosis using non-parametric, non-uniform intensity normalization and support vector machine methods
}

\author{
Mohammad Moghadasi ${ }^{1}$ and Gabor Fazekas ${ }^{2}$
}

\begin{abstract}
Multiple sclerosis (MS) is an inflammatory, chronic, persistent, and destructive disease of the central nervous system whose cause is not yet known but can most likely be the result of a series of unknown environmental factors reacting with sensitive genes. MRI is a method of neuroimaging studies that results in better image contrast in soft tissue. Due to the unknown cause of MS and the lack of definitive treatment, early diagnosis of this disease is important. MRI image segmentation is used to identify MS plaques. MRI images have an image error that is often called non-uniform light intensity. There are several ways to correct non-uniform images. One of these methods is Nonparametric Non-uniform intensity Normalization (N3). This method sharpens the histogram. The aim of this study is to reduce the effect of bias field on the MRI image using N3 algorithm and pixels of MRI images clustered by k-means algorithm. The dimensionality of the data is reduced by Principal Component Analysis (PCA) algorithm and then the segmentation is done by Support Vector Machine (SVM) algorithm. Results show that using the proposed system could diagnose multiple sclerosis with an average accuracy of $\mathbf{9 3 . 2 8 \%}$.
\end{abstract}

Index Terms-Brain MRI Image; Multiple Sclerosis; NonUniform Image; Light Intensity; N3 Method; MR Image Segmentation; Support Vector Machines (SVM); Machine Learning Techniques; K-Means;

\section{INTRODUCTION}

Multiple Sclerosis (MS) is a common, non-traumatic, and neurodegenerative disease that causes young people with disabilities to be characterized pathologically by areas of inflammation, axonal depletion, and the distribution of glycosides throughout the central nervous system. MS often causes sensory, visual, coordination, and other disorders [1]. The two main clinical phenomena of MS are recurrence and progression of the disease. Symptoms of an early recovery in the disease may be due to remodeling, inflammation resolution, and compensatory mechanisms including axonal sodium channel remodeling and membrane flexibility. After repeated attacks the effects of recovery mechanisms are lessened [2]. Early diagnosis of MS is an important step in the treatment process. One of the most important means of diagnosis and follow-up is the use of magnetic resonance imaging (MRI). But with the large volume of MR data being analyzed, it is difficult and time consuming to manually classify these lesions. Auto-

\footnotetext{
${ }^{1,2}$ Department of Informatics, University of Debrecen, Debrecen, Hungary

'E-mail: Mohammad.Moghadasi@inf.unideb.hu

2E-mail: Fazekas.Gabor@inf.unideb.hu
}

immune segmentation of MS lesions has therefore been considered in brain MR images [3]. The human brain is made up of various tissues that can be anatomically divided into sections such as the skull, cerebrospinal fluid, gray matter, white matter, muscle, fat, capillary, and cavity.

MRI is one of the most important models of medical imaging. It is virtually non-invasive and produces excellent contrast for soft tissues. MRI is a great imaging technique for studying the brain. Different MRI protocols are used for this purpose, including the following [6]:

- $\quad$ T1-weighted (T1-W)

- $\quad$ T2-weighted (T2-W)

- PD-weighted (PD-W)

- FLAIR (Fluid-Attenuated inversion recovery T2 images)

Traditional MRI techniques, such as T1-W and T2-W, are very sensitive in detecting MS plaques. Metrics derived from MRI have become a very important paraclinical tool for the diagnosis of MS. Both acute and chronic MS plaques appear as focal areas of high signal intensity in the T2-W sequence. T1$\mathrm{W}$ imaging is very sensitive in detecting inflammatory activity [4]. Brain and spinal cord degeneration is an important part of the pathology of MS and is clinically a major component of disease progression. Quantitative (numerical) criteria for whole-brain atrophy can be obtained by automated and semiautomated methods that demonstrate the progress of brain tissue volume analysis in vivo in a sensitive and reproducible manner. Quantitative analysis of focal lesions in cross-sectional and longitudinal studies is used to calculate the total number of lesions and the total volume of manual and semiautomatic segmental lesions [5]. Brain MRI image segmentation is difficult due to variable imaging parameters, light intensity interference, noise, gradient, motion, echo, and so two steps are generally required before applying any method for segmenting the MS lesion, the removal of image artifacts, and any nonbrain tissue should be removed from the image. MRI images suffer from image error that is often related to the bias field or the intensity of the non-uniformity. The bias field is a lowfrequency, very stable signal that lowers the quality of MRI images, especially those produced by older MRI machines. In most MRI analyzes, bias field correction is a crucial component of the first steps of pre-processing that complicate the effect of automatic image analysis [6]. Bias field correction methods are [7]. 
Segmentation of MRI images to detect multiple sclerosis using non-parametric, non-uniform intensity normalization and support vector machine methods

There are various methods for bias field correction which are divided into three different model groups [9]:

- Model-based methods of production

- Exploration methods

- Combined method

The N3 algorithm is one of the hybrid methods between production-based and exploration-based methods, and the advantage is that it can be applied to any MRI image without prior knowledge of that image [8]. N3 is a non-uniform correction technique that finds a multiplication field that maximizes the frequency content of the scanned intensity distribution [9]. N3 continues with a Gaussian estimation of the actual scan intensity by deconvolution and then using this distribution and the actual scan to obtain the non-uniform field estimation. The field is then cleared by a cubic B-spline light intensity field to estimate the use of the selected distance base point. This filter estimate is then removed from the actual scan and the process restarted. Repeat continues until the uniformity estimates converge [10]. Many preprocessing and fragmentation methods have been introduced for the analysis of MRI images and the detection of MS lesions, which are described below.

In the method of Van Leemput et al., they developed an atlas-based monitoring method and presented an image light intensity-based texture classification using a model derived from the expected value maximization (EM) algorithm. However, simultaneous detection of MS lesions as outliers was not well described by the model. This method derives a prior classification of a digital cerebral atlas containing information about the expected location of White matter (WM), Gray matter (GM) and Cerebrospinal fluid (CSF). Their method also corrects for field MRI heterogeneities, light intensity models, estimating the texture specific image of the data itself, and combining contextual information within a classification using an MRF [11]. In the method of $\mathrm{Wu}$ et al., they presented an automatic segmentation of the MS lesion into three subtypes of KNN-based (k-nearest neighbors) lesion enhancement, black holes, and high-intensity lesions. According to the assumptions of this method, the lesion is only found within the White matter (WM) areas and all lesions are excluded from the mask [12].

The method of Zijdenbos et al., which uses Atlas probability extraction, includes tissue containing White matter (WM), Gray matter (GM) and Cerebrospinal fluid (CSF). Light intensity T1W, T2-W and PD-W images are classified as Artificial Neural Network (ANN) class inputs that segment the lesion [13]. In the method of Shiee et al., it fragments brain tissue in a repetitive manner. They used multi-channel images to segment the main structure of the whole brain. They Used atlas-based segmentation techniques that applied statistical atlas and topological atlas together with the Fuzzy C-Means (FCM) algorithm for segmentation. As reported by them, the advantages of topological atlas are that all fragmented structures are location-bound, which allows subsequent processes to resuscitate and detect the membrane $[14,15]$. Seld et al., presented a new (N3) method of correction for non- uniformity of image light intensity, did not rely on a parametric model of tissue light intensity or lesion fragmentation within continuous areas. The former is illustrated by the ability of the N3 method to correct the non-uniformity of image light intensity in MRI data irrespective of the pulse sequence without initial training. The other is illustrated by the ability of methods to correct nonuniformity by using the structure of a random field even when nonuniformity is not clearly visible. N3 is a fully automated iterative method that operates on $3 \mathrm{D}$ volumetric data $[16,17]$. In Borys et al. method, an approach of correcting the intensity uniformity is presented. The idea was to replace Wenn's FC-harmon method with the K-harmonic method. The algorithm was tested with MRI datasets obtained from a phantom object using a breast MRI coil to simulate real conditions during the study. The results were compared with the other five methods using two indices - integral uniformity and standard deviation of the signal within the object. For the proposed and improved method, the least integral uniformity and reasonable signal deviation were obtained [18]. With Lin et al. method, the proposed algorithm first applies N3, then follows Fuzzy C-Means (FCM), and then smooths the bias field created using the Gaussian kernel and the "B - spline" surface junctions to change the texture contrast problem. The results of segmentation based on N3 FCM-corrected images were compared with N3 and FCM-modified images alone and, alternatively, Coherent Local Intensity Clustering (CLIC), corrected images. The quality of segmentation was evaluated and rated by radiologists based on different correction methods [19, 20, 21].

In this paper, we aim to improve the existing methods by applying N3 algorithm as one of the pre-processing steps of MR images and bias field deletion in segmentation of MRI images.

\section{NON-UNIFORMITY OF IMAGE LIGHT INTENSITY}

Non-uniformity of image light intensity makes the light intensity change slow and it is often seen in MR images due to some of the following factors [22]:

- Induction of radio frequency (RF).

- Non-uniform heterogeneous receiver sensitivity

- Electrodynamic interactions with the purpose described as RF diffusion

- Wave effect mode

Other less important factors involved in non-homogeneity include:

- Eddy currents caused by switching the slope of the field

- Coil disturbance

- RF data bandwidth filter

- Geometric distortion

The effect of geometric distortion on the non-uniformity of image light intensity for normal clinical scanners can be ignored. Since the grading routines often represent several millimeters of surface geometric distortion on a field of one side size, and the distortions of the squares change slowly, then one 
Segmentation of MRI images to detect multiple sclerosis using non-parametric, non-uniform intensity normalization and support vector machine methods

can expect the corresponding changes in image light intensity to be one percent. This is less than the other causes of malignancy $[23,24,25]$. In MR, the spatial imaging process is encoded by resonant frequency. Hence, the frequencydependent changes in the sensitivity of the RF reception system result in the corresponding light intensity changes in the image results along the frequency coding path. If a filter is used to limit the bandwidth of an incoming signal that is non-uniform frequency response, a similar problem arises. Since the sensitivity of an RF coil is often uniform and close to its resonance frequency and the coil resonance frequency of the inner subject, the uniformity of image light intensity is improved by adjusting the coil for MR resonance frequency before each scan. Therefore, light intensity changes can disrupt RF coils and filter the data bandwidth that can be considered a scanner defect [26].

\section{A. Non-uniformity model}

The correction problem for non-uniformity of image light intensity is greatly simplified if modeled as a fine multiplier. This model is consistent with the multiplicative non-uniformity caused by differences in the sensitivity of the coil of acceptance and the small non-uniformity amplitude due to the excitation and excitation currents. Consider the following model of image construction in MR [27, 28, 29]:

$$
v(x)=u(x) f(x)+n(x)
$$

In $\mathrm{x}$ location, $v$ is the measured signal, $\mathrm{u}$ is the actual signal propagated from the tissue, $f$ is a non-random variable bias field, and $n$ is the white Gaussian noise assumed to be independent of $u$. The difficulty of compensating for the nonuniformity of the image light intensity is to estimate $f$. A combination of multipliers and additives makes this difficult. Consider a non-noise case where the true light intensity $u$ at each axis of the X-location is independent of randomly distributed random variables. Then by taking the logarithm as $\hat{u}(x)=\log (u(x))$ Let the model of image construction be summed up:

$$
\hat{v}(x)=\hat{u}(x)+\hat{f}(x)
$$

Now we show the probable density of $\hat{v}, \hat{u}$ and $\hat{f}$ with $\mathrm{V}, \mathrm{U}$ and $\mathrm{F}$, respectively. Assuming that $\hat{f}$ and $\hat{u}$ are independent of each other, the random variables are unrelated, their total distribution can be found by deconvolution:

$$
V(\hat{v})=F(\hat{v}) U(\hat{v})=\int F(\hat{v}-\hat{u}) U(\hat{u}) d \hat{u}
$$

The non-uniform distribution of $\mathrm{F}$ can be seen as an opaque distribution of light intensity [17]. From the signal processing point of view, blurring results in a reduction in the high frequency component $U$ field. The non-uniform correction of the light intensity of the image is to return the frequency of the
U contents. Since the shape of the F-core is not known, it is unclear what frequency components of $U$ can be retrieved to obtain the observed $\mathrm{V}$ distribution from the actual $\mathrm{U}$ distribution. However, since the uniformity of the field $\backslash$ hat $\{\mathrm{f}\}$ is limited to the soft variations, there are several possible $U$ distributions corresponding to the given $\mathrm{V}$ distribution. In $\mathrm{N} 3$, the non-uniformity correction method is to find smooth changes in the multiplicative field so as to maximize the $\mathrm{U}$ frequency.

\section{B. Non-uniformity correction methods}

The methods available to correct for non-uniformity of image light intensity can be classified into three groups: analytical non-uniformity modeling, modified proprietary protocols that measure non-uniformity, and data-driven post processing. Suppose a multiplicative non-uniform field is corrected by dividing it from the image. The analytical methods described above are useful tools for understanding the mechanism of the generation of changes in image light intensity. However, the non-uniform dependence on the geometry makes these methods impractical because a new model is required for each scan. Among the techniques that include modified proprietary protocols, the fact is that inhomogeneity is largely subject-specific and is an area for excluding them that involves regular scanning of a calibration phantom. In addition, repeated scans of the calibration phantoms in the clinical setting are not practical [30]. The most common data-driven post processing methods are used for homomorphic filtering to estimate multiplicative non-uniform field and image segmentation. These methods assume that the frequency content of the non-uniform field is less than the anatomy $[17,31,32]$.

\section{THE PROPOSED METHOD}

In the first step, the preprocessing step is performed to remove the skull, and then the N3 algorithm is performed to remove the noise from the image. By executing this step, the corrected image is applied as input to the K-means algorithm. We consider the number of clusters to be equal to four, because there are four different tissue types: Gray matter (GM), White matter (WM), Cerebrospinal fluid (CSF) and background in the image after clustering the result. The vector form is given as an input to the principal component analysis (PCA) algorithm for feature recognition and limiting search space. Then the output of this step is applied to the SVM algorithm for classification and afterwards clustered by KNN. Ultimately with an unintentional distribution, $70 \%$ of the data randomly was applied to training and 30\% test to determine whether a person is healthy or unhealthy.

\section{A. Practical Implementation of Method N3}

Let's assume if " $d$ " $=\left(d_{1}, d_{2}, \ldots, d_{N}\right)^{T}$ the light intensity, "a" the wavelength of an MRI scan, and " $b "=\left(b_{1}, b_{2}, \ldots, b_{N}\right)^{T}$ the corresponding gains result in the bias field. As it has been generally done in previous articles by bias field correction $[8$, 24-26].The N3 method assumes that $d$ and $b$ are logarithmic, so that the effect of $b$ is incremental. The $b$ is assumed to have a shape with zero Gaussian mean and a definite variance. Histogram d is also a blurred version of the actual histogram, 
Segmentation of MRI images to detect multiple sclerosis using non-parametric, non-uniform intensity normalization and support vector machine methods

and the background picture leads to a convolution with histogram $b$. The purpose of the algorithm will be to reverse the N3 method by deconvolution as well as by estimating the smoothed bias field model. This inversion process is repeated continuously to improve the bias field estimates [33].

\section{B. Deconvolution step}

The first step in the deconvolution algorithm is the histogram. By estimating the current bias field represented by $\tilde{b}$, a normalized histogram with $d-\tilde{b}$ is calculated from the corrected bias field data $d-\tilde{b}$, and assumed to be a soft bias field in the first iteration $(\tilde{b}=0)[34,35]$. The bin centers are given by the following formulas:

$$
\begin{aligned}
& \tilde{\mu}_{1}=\min (d-\tilde{b}) \\
& \tilde{\mu}_{K}=\max (d-\tilde{b}) \\
& \tilde{\mu}_{k}=\tilde{\mu}+(k-1) h
\end{aligned}
$$

Which $h=\left(\tilde{\mu}_{k}-\tilde{\mu}_{1}\right) /(k-1)$ is bin width. $\left(v_{k}, k=\right.$ $1, \ldots, K)$ histogram entries are filled using the interpolation model [10]:

$$
v_{k}=\frac{1}{N} \sum_{1=1}^{N} \varphi\left[\frac{d_{i}-\tilde{b}_{i}-\tilde{\mu}_{k}}{h}\right], \varphi[s]= \begin{cases}1-|s| & \text { if }|s|<1 \\ 0 & \text { otherwise }\end{cases}
$$

By defining $\hat{v}$ as a 512-dimensional vector such that $\hat{v}=$ $\left(v_{1}, v_{2}, \ldots, v_{k}\right)^{T}$, the histogram is deconvolved by the following formula:

$$
\hat{\pi}=F^{-1} D F \hat{v}
$$

In the above formula $\mathrm{F}$ represents the discrete Fourier transform matrix with the following elements:

$$
F_{n, k}=e^{-2 \pi j(k-1)(n-1) / 512}, n, k=1, \ldots, 512
$$

And D represents a diagonal matrix with the following elements:

$$
D_{k}=\frac{f_{k}^{*}}{\left|f_{k}^{*}\right|^{2}+\gamma}, k=1, \ldots, 512
$$

Where $\gamma=0.1$ is a constant value and $f=$ $\left(f_{1}, f_{2}, \ldots, d_{512}\right)^{T}=F g$ where $g$ is a 512 vector and contains the hidden Gaussian kernel with the following variance:

$$
\sigma^{2}=\frac{f^{2}}{8 \log 2}
$$

\section{Bias correction step}

When the $\hat{\pi}$ histogram is decanulated, the light intensity of the corresponding $\tilde{d}_{\mu l}$ corrected image in the decanogram histogram at each bin center of $\tilde{\mu}_{l}, l=1, \ldots, K$ is estimated by the following formula [10]:

$$
\tilde{d}_{\mu l}=\sum_{k} w_{k}^{l} \tilde{\mu}_{k}, w_{k}^{l}=\frac{\left(\tilde{\mu}_{l} \mid \tilde{\mu}_{k}, \sigma_{k}^{2}\right) \hat{\pi}_{k}}{\sum_{k}\left(\tilde{\mu}_{l} \mid \tilde{\mu}_{k}, \sigma_{k}^{2}\right) \hat{\pi}_{k}}
$$

And the magnitude of the DD-corrected image is found in each voxel with linear interpolation:

$$
\tilde{d}_{i}=\sum_{l=1}^{K} \tilde{d}_{\mu l} \varphi\left[\frac{d_{i}-\tilde{b}_{i}-\tilde{\mu}_{k}}{h}\right], \varphi[s]= \begin{cases}1-|s| & \text { if }|s|<1 \\ 0 & \text { otherwise }\end{cases}
$$

Finally, the remaining $r=d-\tilde{d}$ is calculated and cleared to obtain the bias field estimate:

$$
b_{i}=\Phi \tilde{c}
$$

Calculated in the previous formula $\tilde{c}$ by the following:

$$
\tilde{c} \leftarrow\left(\Phi^{T} \Phi+\mathrm{N} \beta \psi\right)^{-1} \Phi^{T} \mathrm{r}
$$

Which in this $\Phi$ formula is an $N \times M$ matrix of spatial smoothing basic functions, where each $\Phi_{i, m}$ element, $\mathrm{m}$ th Evaluates the base function in the i voxel. $\psi$ is a semiconductor positive matrix that fines the curvature of the bias field, and the $\beta$ is a user-defined adjustment constant whose default value is $\beta=10^{-7}$.

\section{POST-PROCESSING}

The N3 method is repeated alternately between the deconvolution phase and the bias field correction step until the standard deviation of the bias estimation difference between the two iterations is less than a specified threshold. By default, the N3 method operates on a sub-volume. After convergence, the estimation of the bias field exponentially returns to the amplitude of the original image, which subsequently corresponds to Equation 13 and, for example, to $r=\exp (\tilde{b})$. The resultant coefficients are then used to calculate the final estimate of the bias field by evaluating from Equation 12 with $\Phi$ full resolution. Finally, inaccurate data are segmented by estimating the bias field to obtain the corrected volume.

\section{RESULTS}

Two databases were used in this article, one consisting of brain MRI images of healthy individuals and the other one belonging to patients who were all infected with MS. The first database was obtained from the CASI Laboratory of Surgery at the University of North Carolina. This database identifies people who have had a mild or severe stroke or who have had any brain injury for the probability of error in the test results in 
Segmentation of MRI images to detect multiple sclerosis using non-parametric, non-uniform intensity normalization and support vector machine methods

red and are recommended to be eliminated if necessary. The second database is obtained from the eHealth lab of Computer Science University of Cyprus. All images are sequenced for each individual, and we selected a specific cross-section where the highest level of the brain was visible and changed the TIFF format to JPG to run the program quickly. This database contains the date of birth of all patients, date of shooting, age and folder name of each individual.

Skull removal is one of the important steps in preprocessing because fat, skull and other non-brain tissues can be a cause of incorrect classification in some ways due to the similarity of the light intensity of the image to brain structures.
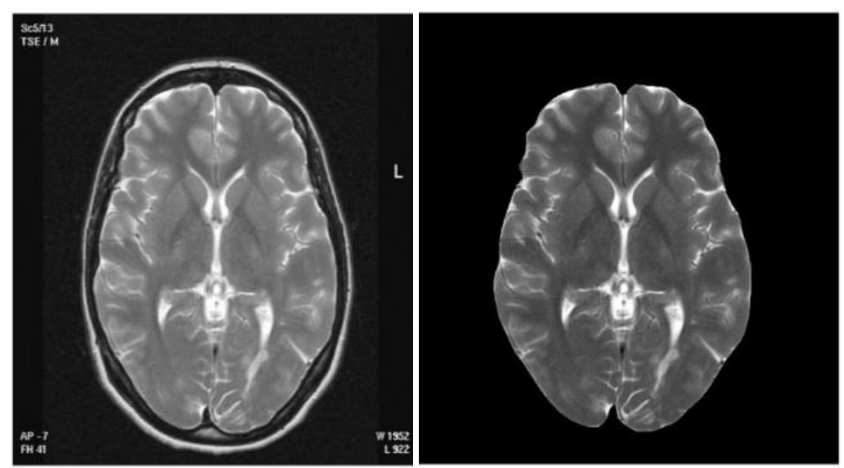

Fig. 1. Preprocessed image sample and skull removal

After executing various steps of $\mathrm{N} 3$ algorithm, its output is as follows:

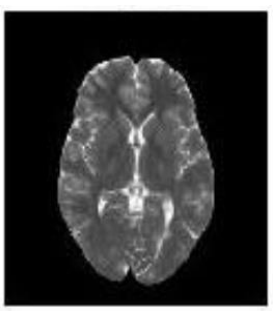

Original

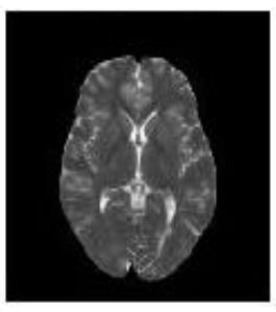

Corrected

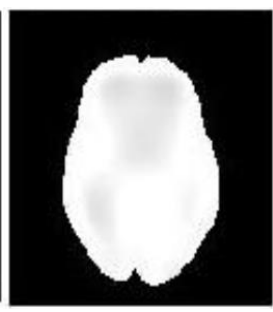

Estimated bias Field
Fig. 2. Approximate images, inputs, outputs, and bias fields of N3 algorithm

The output of the previous step is considered as the input of this step. In this method, after finding the appropriate $\mathrm{K}$ number to find the optimal state, it is usually considered $\mathrm{k}=4$ with the number of different brain tissues including GM, White matter (WM), cerebrospinal fluid, and background. In order to evaluate the accuracy of the proposed method in the appropriate area of MRI images we use:

- Method 1: Accuracy of the proposed method in correct diagnosis of MS disease using N3

- Method 2: Accuracy of the proposed method in correct diagnosis of MS without using N3

You can see the disaggregation matrix for the proposed model using Support Vector Machine (SVM) and both methods 1 and 2 in Table.
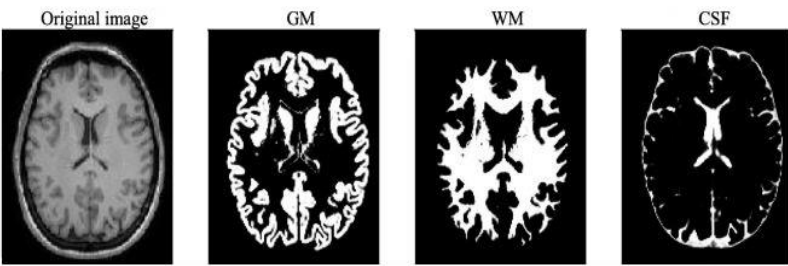

Fig. 3. Output from clustering of human brain MRI image with 4 clusters

To evaluate the N3 method, we compare our proposed method with that of the N3 algorithm, and we show that with age we have improved the algorithm by $1.5 \%$ compared to the non-N3 algorithm. By repeatedly replicating the above method and applying these algorithms to the images in the database randomly and obtain the average percentage improvement of N3 method.

TABLE I: Compare with and without N3 methods using SVM

\begin{tabular}{|c|c|}
\hline Algorithm & Average correct diagnosis \\
\hline correct diagnosis of MS disease using N3 & 93.28 \\
\hline correct diagnosis of MS without using N3 & 89.5 \\
\hline
\end{tabular}

\section{DISCUSSION}

N3 stands for Non-Parameter Normalization of NonParametric Image Intensity and is a new data-driven method for correcting non-uniformity of image light in MRI data. In this paper, a novel method of classifying machine learning-based MS disease using datasets is presented. The proposed method is performed in five steps:

- Calling images of healthy and unhealthy patients from the database

- Apply pre-processing step on images

- Run N3 algorithm and remove bias field error from images

- Input Implementation of the K-means algorithm for clustering the output from the previous step

- Run SVM algorithm to classify existing images

The proposed method used the database information mentioned above. By using N3 algorithm the bias field effect of the image is minimized and then clustered by k-means algorithm and then by PCA algorithm the dimensionality of the data is reduced and then the segmentation is done by Support Vector Machine (SVM) algorithm and the images are the range is normalized from 0 to 255 , and finally, based on these features, images and lesions are classified using the SVM algorithm. Experimental results showed that using the proposed system, one could diagnose multiple sclerosis with an average accuracy of $93.28 \%$. 
Segmentation of MRI images to detect multiple sclerosis using non-parametric, non-uniform intensity normalization and support vector machine methods

\section{REFERENCES}

[1]. Mohammad Moghadasi and Gabor Fazekas, "Multiple sclerosis Lesion Detection via Machine Learning Algorithm based on converting 3D to 2D MRI images", Infocommunications Journal, Vol. XII, No 1, March 2020, pp. 38-44. DoI:10.36244/ICJ.2020.1.6

[2] Mohammad Mohammad Moghadasi and Dr. Gabor Fazekas, "An Automatic Multiple Sclerosis Lesion Segmentation Approach based on Cellular Learning Automata" International Journal of Advanced Computer Science and Applications(IJACSA), 10(7), 2019. DoI: 10.14569/IJACSA.2019.0100726

[3] P. Baranyi, A. Csapo and G. Sallai: Cognitive Infocommunications (CogInfoCom), Springer International Publishing, 2015, Springer International Publishing, Switzerland, p. 191 (978-3-319-19607-7), DOI: 10.1007/978-3-319-19608-4

[4] L. Kovács, Gy. Eigner: “Tensor Product Model Transformation-based Parallel Distributed Control of Tumor Growth", Volume 15, Issue Number 3, 2018. DoI: 10.12700/APH.15.3.2018.3.7

[5] Csapó, Gábor \& Csernoch, Maria \& Abari, Kálmán., "Sprego: case study on the effectiveness of teaching spreadsheet management with schema construction. Education and Information Technologies", Education and Information Technologies, 2019, DoI: $10.1007 / \mathrm{s} 10639-019-10024-2$

[6] M. Moghadasi and G. Fazekas, "Multiple Sclerosis Detection via Machine Learning Algorithm, Accurate Simulated Database 3D MRI to 2D Images, using value of Binary Pattern Classification - A Case Study," 2019 10th IEEE International Conference on Cognitive Infocommunications (CogInfoCom), Naples, Italy, 2019, pp. 233240, DoI: 10.1109/CogInfoCom47531.2019.9089962

[7] Zhang, Yudong, Siyuan Lu, Xingxing Zhou, Ming Yang, Lenan Wu, Bin Liu, Preetha Phillips, and Shuihua Wang.: "Comparison of Machine Learning Methods for Stationary Wavelet Entropy-Based Multiple Sclerosis Detection: Decision Tree, k-Nearest Neighbors, and Support Vector Machine." SIMULATION 92, no. 9 (September 2016): 861-71. DoI: 10.1177/0037549716666962

[8] Coupland AP, Thapar A, Qureshi MI, Jenkins H, Davies AH.: "The definition of stroke" Journal of the Royal Society of Medicine. 2017 Jan; 110(1):9-12. DoI: 10.1177/0141076816680121

[9] C. Li, J. C. Gore, and C. Davatzikos, "Multiplicative intrinsic component optimization (MICO) for MRI bias field estimation and tissue segmentation," Magnetic resonance imaging, vol. 32, no. 7, pp. 913-923, 2014. DoI: 10.1016/j.mri.2014.03.010

[10] C. T. Larsen, J. E. Iglesias, and K. Van Leemput, "N3 bias field correction explained as a Bayesian modeling method," in Bayesian and graphical models for biomedical imaging: Springer, 2014, pp. 1-12. DoI: 10.1007/978-3-319-12289-2_1

[11] Mohammad Moghadasi, Seyed Majid Mousavi and Gábor Fazekas, "Cloud Computing Auditing" International Journal of Advanced Computer Science and Applications(ijacsa), 9(12), 2018. DoI: 10.14569/IJACSA.2018.091265

[12] Boyes, R. G., Gunter, J. L., Frost, C., Janke, A. L., Yeatman, T., Hill, D. L. G., Bernstein, M. A., Thompson, P. M., Weiner, M. W., Schuff, N., Alexander, G. E., Killiany, R. J., DeCarli, C., Jack, C. R., \& Fox, N. C. (2008). Intensity non-uniformity correction using N3 on 3-T scanners with multichannel phased array coils. NeuroImage, 39(4), 1752-1762. DoI: 10.1016/j.neuroimage.2007.10.026

[13] X. Lladó et al., "Segmentation of multiple sclerosis lesions in brain MRI: a review of automated approaches," Information Sciences, vol. 186, no. 1, pp. 164-185, 2012. DoI: 10.1007/s00234-011-0992-6

[14] K. Van Leemput, F. Maes, D. Vandermeulen, A. Colchester and P. Suetens, "Automated segmentation of multiple sclerosis lesions by model outlier detection," in IEEE Transactions on Medical Imaging, vol. 20, no. 8, pp. 677-688, Aug. 2001, DoI: 10.1109/42.938237
[15] Y. Wu et al., "Automated segmentation of multiple sclerosis lesion subtypes with multichannel MRI," NeuroImage, vol. 32, no. 3, pp. 1205-1215, 2006. DoI: 10.1007/s00234-011-0886-7

[16] Zhang, Yudong, Siyuan Lu, Xingxing Zhou, Ming Yang, Lenan Wu, Bin Liu, Preetha Phillips, and Shuihua Wang.: "Comparison of Machine Learning Methods for Stationary Wavelet Entropy-Based Multiple Sclerosis Detection: Decision Tree, k-Nearest Neighbors, and Support Vector Machine." SIMULATION 92, no. 9 (September 2016): 861-71. DOI: 10.1177/0037549716666962

[17] Coupland AP, Thapar A, Qureshi MI, Jenkins H, Davies AH.: "The definition of stroke" Journal of the Royal Society of Medicine. 2017 Jan; 110(1):9-12. DoI: 10.1177/0141076816680121

[18] S. M. Mousavi, M. Moghadasi and G. Fazekas, "Dynamic resource allocation using combinatorial methods in Cloud: A case study," 2017 8th IEEE International Conference on Cognitive Infocommunications (CogInfoCom), Debrecen, 2017, pp. 000073-000078, DoI: 10.1109/CogInfoCom.2017.8268219

[19] C. Confavreux, S. Vukusic, T. Moreau, and P. Adeleine, "Relapses and progression of disability in multiple sclerosis," New England Journal of Medicine, vol. 343, no. 20, pp. 1430-1438, 2000. DoI: 10.1056/NEJM200011163432001

[20] A. Ortiz, J. Górriz, J. Ramírez, D. Salas-Gonzalez, and J. M. LlamasElvira, "Two fully-unsupervised methods for MR brain image segmentation using SOM-based strategies," Applied Soft Computing, vol. 13, no. 5, pp. 2668-2682, 2013. DoI: 10.1016/j.asoc.2012.11.020

[21] A. Y. Sharma and Y. K. Meghrajani, "Automated segmentation of multiple sclerosis lesions using statistical approach," 2015 International Conference on Innovations in Information, Embedded and Communication Systems (ICIIECS), Coimbatore, India, 2015, pp. 1-5, DoI: 10.1109/ICIIECS.2015.7193144

[22] J. Bogaert, S. Dymarkowski, A. M. Taylor, and V. Muthurangu, Clinical cardiac MRI. Springer Science \& Business Media, 2012. DoI: 10.1007/978-3-642-23035-6

[23] Failo R, Wielopolski PA, Tiddens HA, Hop WC, Mucelli RP, Lequin MH. Lung morphology assessment using MRI: a robust ultra-short TR/TE 2D steady state free precession sequence used in cystic fibrosis patients. Magn Reson Med. 2009 Feb;61(2):299-306. DOI: 10.1002/mrm.21841. PMID: 19165879

[24] Hickman, S J, G J Barker, P D Molyneux, and D H Miller. “Technical Note: The Comparison of Hypointense Lesions from 'Pseudo- T1' and T1-Weighted Images in Secondary Progressive Multiple Sclerosis.” Multiple Sclerosis Journal 8, no. 5 (October 2002): 433-35. DoI: 10.1191/1352458502ms824xx

[25] M. N. Ahmed, S. M. Yamany, N. Mohamed, A. A. Farag and T. Moriarty, "A modified fuzzy c-means algorithm for bias field estimation and segmentation of MRI data," in IEEE Transactions on Medical Imaging, vol. 21, no. 3, pp. 193-199, March 2002, DoI: $10.1109 / 42.996338$

[26] Lin, M., Chan, S., Chen, J. H., Chang, D., Nie, K., Chen, S. T., Lin, C. J., Shih, T. C., Nalcioglu, O., \& Su, M. Y. (2011). A new bias field correction method combining N3 and FCM for improved segmentation of breast density on MRI. Medical physics, 38(1), 5-14. DOI: $10.1118 / 1.3519869$

[27] A. P. Zijdenbos, R. Forghani and A. C. Evans, "Automatic "pipeline" analysis of 3-D MRI data for clinical trials: application to multiple sclerosis," in IEEE Transactions on Medical Imaging, vol. 21, no. 10, pp. 1280-1291, Oct. 2002, Dor: 10.1109/TMI.2002.806283

[28] Sweeney, E. M., Shinohara, R. T., Shiee, N., Mateen, F. J., Chudgar, A. A., Cuzzocreo, J. L., Calabresi, P. A., Pham, D. L., Reich, D. S., \& Crainiceanu, C. M. (2013). OASIS is Automated Statistical Inference for Segmentation, with applications to multiple sclerosis lesion segmentation in MRI. NeuroImage. Clinical, 2, 402-413. DOI: $10.1016 /$ j.nicl.2013.03.002 
Segmentation of MRI images to detect multiple sclerosis using non-parametric, non-uniform intensity normalization and support vector machine methods

[29] Shiee, N.,Bazin, P.L., Ozturk, A., Reich, D. S.,Calabresi,P. A., \& Pham, D. L. (2010). A topology-preserving approach to the segmentation of brain images with multiple sclerosis lesions. NeuroImage, 49(2), 1524-1535. Dor: 10.1016/j.neuroimage.2009.09.005

[30] Sled JG, Zijdenbos AP, Evans AC. A nonparametric method for automatic correction of intensity nonuniformity in MRI data. IEEE Trans Med Imaging. 1998 Feb;17(1):87-97. Dor: 10.1109/42.668698. PMID: 9617910

[31] Vrooman, H. A., Cocosco, C. A., van der Lijn, F., Stokking, R., Ikram, M. A., Vernooij, M. W., Breteler, M. M. \& Niessen, W. J. (2007). Multi-spectral brain tissue segmentation using automatically trained k-Nearest-Neighbor classification. NeuroImage, 37(1), 71-81. DoI: 10.1016/j.neuroimage.2007.05.018

[32] Kwan RK, Evans AC, Pike GB. MRI simulation-based evaluation of image-processing and classification methods. IEEE Trans Med Imaging. 1999 Nov;18(11):1085-97. DOI: 10.1109/42.816072. PMID: 10661326.

[33] Collins DL, Zijdenbos AP, Kollokian V, Sled JG, Kabani NJ, Holmes CJ, Evans AC. Design and construction of a realistic digital brain phantom. IEEE Trans Med Imaging. 1998 Jun;17(3):463-8. PMID: 9735909. DoI: $10.1109 / 42.712135$

[34] J. Grande-Barreto and P. Gómez-Gil, "Unsupervised brain tissue segmentation in MRI images," 2018 IEEE International Autumn Meeting on Power, Electronics and Computing (ROPEC), Ixtapa, Mexico, 2018, pp. 1-6, Dor: 10.1109/ROPEC.2018.8661425

[35] Lötsch, J., Schiffmann, S., Schmitz, K. et al. "Machine-learning based lipid mediator serum concentration patterns allow identification of multiple sclerosis patients with high accuracy", Sci Rep 8, 14884 (2018). DOI: $10.1038 / \mathrm{s} 41598-018-33077-8$

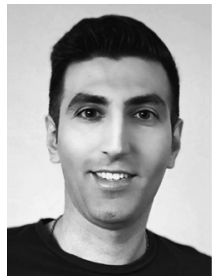

Mohammad Moghadasi is a $\mathrm{PhD}$ student at University of Debrecen, Faculty of Informatics, with a backgorund of Software Information Technology. $\mathrm{He}$ is currently working in digital image processing on multiple sclerosis lesion detection. He is interested and has experience in database, networking and cloud computing as well. He is also a lecturer.

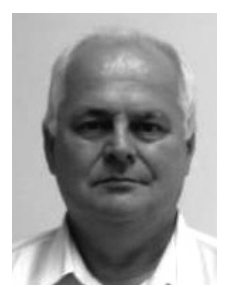

Dr. Gabor Fazekas is an Associate Professor at University of Debrecen, Faculty of Informatics, Department of Information Technology. He has extensive experience in supervising $\mathrm{PhD}$ dissertations. $\mathrm{He}$ is very well knowledged in reeasrch and teaching. $\mathrm{He}$ recently was retired but still supervising $\mathrm{PhD}$ students in the fields of computer systems, databases, image processing, combinatorial coding, didactic and many more.

\section{DECLARATIONS}

Funding

This research did not receive any specific grant from funding agencies in the public, commercial, or not-for-profit sectors. This work was not financially supported by any organization. 